\title{
Heart Block in the Patients with COVID-19
}

${ }^{1}$ Md Ripon Ahammed, MBBS, ${ }^{1}$ Medha Sharath, MBBS, ${ }^{1}$ Mehul Sinha, MBBS, ${ }^{1}$ Cristina Sestacovschi, MD, ${ }^{1}$ Varadha Retnakumar, MBBS, ${ }^{1}$ Naisargee Solanki, MBBS

${ }^{1}$ Research and Academic Affairs, Larkin Community Hospital, South Miami, Florida, USA

\section{$\underline{\text { Abstract }}$}

Background: Since the emergence of the SARS COV-19 pandemic, multiple extrapulmonary manifestations of the virus have been reported from around the world. Cardiovascular complications including arrhythmias in patients with COVID-19 have been described in multiple studies. Our aim was to review various case reports detailing the new onset of heart block in COVID-19 patients and to summarise the clinical course of these patients.

Methods: We systematically reviewed all reports published and indexed in PubMed, Scopus, and Embase between March 2020 to May 2021, analyzing the relation between the demographics of the patients, pre-existing comorbidities, and the progression of heart block in patients infected with COVID-19.

Results: We identified and included in this study 30 relevant articles describing 49 COVID-19 patients with heart block. Among them, 69.3\% $(n=34)$ of patients suffered from at least one comorbidity. $36.73 \%(\mathrm{n}=18)$ of the patients showed spontaneous resolution of the heart block. Conversely, $63.26 \%(n=31)$ of the patients had persistent heart block, out of which $16.33 \%(n=8)$ and $42.86 \%(\mathrm{n}=21)$ were implanted with a temporary and permanent pacemaker respectively. The reported mortality rate was $22.45 \%(\mathrm{n}=11)$ during hospitalization. We noted that $45.45 \%$ $(n=5)$ of the patients who died had complete heart block. $24.49 \%(n=12)$ of the patients in the studies we reviewed were suspected of having myocarditis. However, none were confirmed with MRI or cardiac biopsy.

\section{Conclusions:}

Additional research is necessary to unearth the mechanism of development of heart block in COVID-19 patients as well as its implications on the clinical course and prognosis. Physicians must be aware of the importance of monitoring patients hospitalized for COVID-19 for arrhythmias including heart blocks, especially in the presence of comorbidities. Early detection can improve the prognosis of the patient.

Keywords: Heart Block; Arrhythmia; COVID-19; SARS-CoV-19; Heart 


\section{Background:}

Since the first reports of atypical pneumonia in Wuhan province of China, the coronavirus disease 2019 (COVID-19) has evolved into a major world health issue. Although pneumonia is the most significant manifestation of severe acute respiratory syndrome coronavirus-2 (SARSCoV-2) infection', a range of extra-pulmonary manifestations have been reported, such as venous thromboembolism, cardiac complications, gastrointestinal abnormalities, acute kidney insult, hepatocellular injury, hyperglycemic state, neurological illnesses and dermatological complications $^{2}$. $14.1 \%$ of hospitalized COVID-19 cases have been reported to develop cardiovascular abnormalities which mainly include acute coronary syndrome, arrhythmia, and acute heart failure ${ }^{3}$; although patients with fulminant myocarditis, pericarditis, cardiac tamponade, Takotsubo cardiomyopathy, and cor pulmonale have also been described ${ }^{4-7}$. It is recognized that cardiac involvement in COVID-19 leads to a worse prognosis ${ }^{8}$. Ventricular arrhythmias, atrial arrhythmias, sinus bradycardia, heart block, ST-segment changes, and prolonged QTc have all been reported in COVID-19 patients. Meta-analyses have estimated the arrhythmia incidence rate in patients hospitalized for COVID-19 to be from $6.9 \%$ to $10.3 \%{ }^{9,10}$.

Heart block is the least documented form of arrhythmia that is seen in COVID-19 patients ${ }^{10}$ and the incidence, nature, and impact of this type of arrhythmia among COVID-19 patients is yet to be studied. Hence, the aim of this systematic review is to understand potential patterns of heart block, association with pre-existing comorbidities, and the effect on the clinical course including the outcome of heart block in this group of patients.

\section{METHODS}

\section{Literature Search}

2 researchers (RA, and MS) independently conducted the search and selection of the studies. We included case reports and case series published from March 2020 to May 2021. The systematic search was carried out in May 2021 utilizing PubMed (Medline), Scopus, and Embase. The keywords that we searched for include: heart block OR arrhythmia OR dysrhythmia OR bradyarrhythmia OR brady-arrhythmia OR atrioventricular block OR atrioventricular block AND novel coronavirus OR new coronavirus OR nCoV OR Cov-2 OR Cov2 OR COVID AND case OR case report OR case reports OR case series.

\section{Screening, Inclusion and Exclusion Criteria}

There was no pre-specified exposure/ intervention, comparison groups, or outcomes as we intended to summarize all available case reports of COVID-19 and heart block. Titles, abstracts, and full-texts were screened by RA and MS independently. Letters to the editor, commentaries, systematic reviews, meta-analyses, and duplicates were excluded. Figure 1 is the PRISMA flow diagram which shows the selection process of our study. Our search criteria yielded the identification of 437 articles. 407 articles were excluded after screening. 2 articles were further 
medRxiv preprint doi: https://doi.org/10.1101/2022.01.05.22268779; this version posted January 5, 2022. The copyright holder for this preprint (which was not certified by peer review) is the author/funder, who has granted medRxiv a license to display the preprint in perpetuity.

It is made available under a CC-BY 4.0 International license.

excluded after review since they did not describe heart block or COVID-19, and 1 article was excluded since it was a systematic review. 3 articles were added after a manual search. Finally, 30 eligible articles were selected for our systematic review.

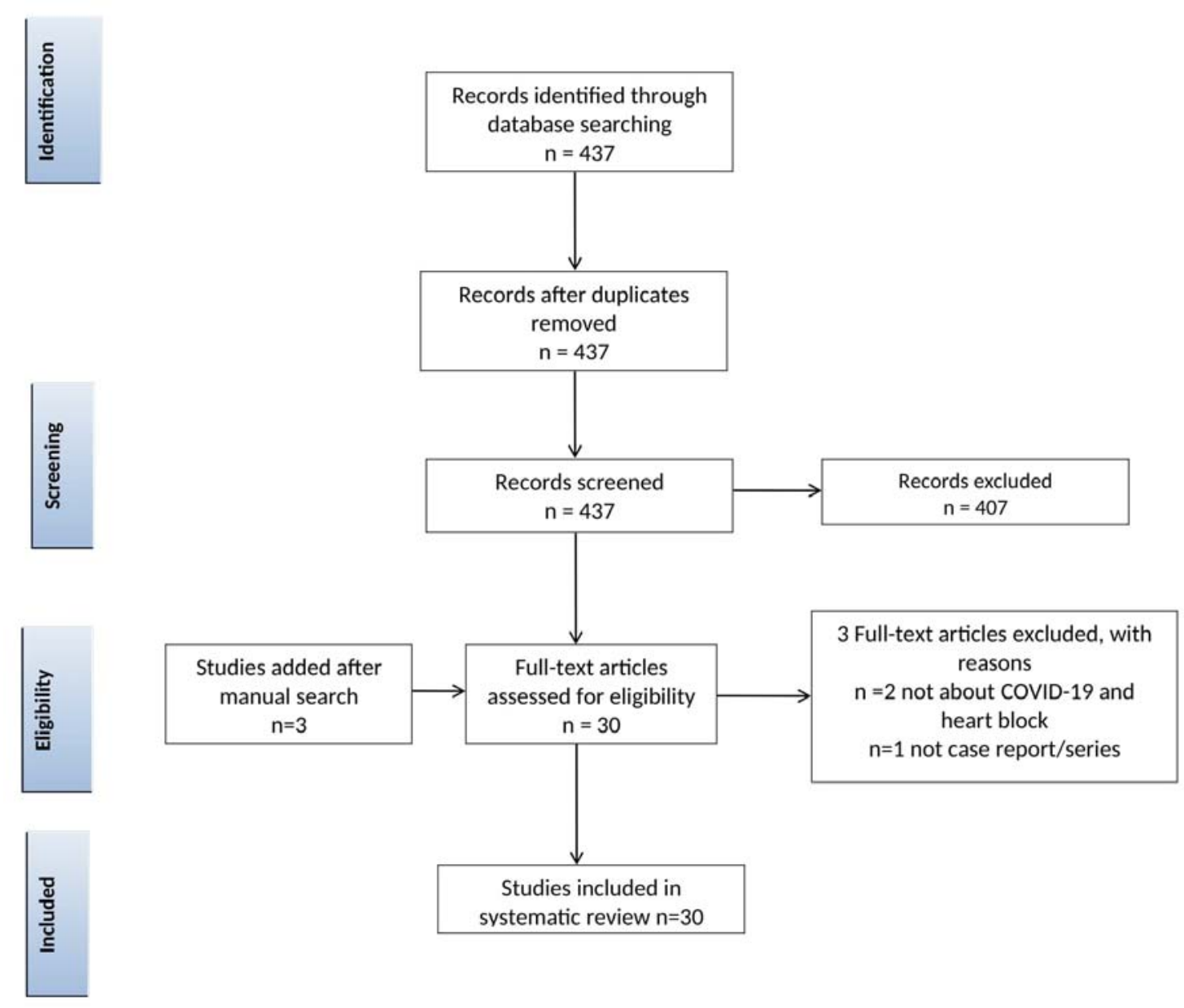


medRxiv preprint doi: https://doi.org/10.1101/2022.01.05.22268779; this version posted January 5, 2022. The copyright holder for this preprint (which was not certified by peer review) is the author/funder, who has granted medRxiv a license to display the preprint in perpetuity.

It is made available under a CC-BY 4.0 International license .

\section{Extraction of Data}

Data from included studies were extracted by reviewers and verified by using a standard form. Data regarding patient demographics; comorbidities; medications; baseline ECG abnormalities; laboratory findings; onset, duration, characteristics, and resolution of heart block; as well as details of pacemaker implant were collected in an excel sheet. The data was verified twice to check for any inaccuracies or discrepancies.

\section{RESULTS}

We identified and included in this study 30 relevant articles describing 49 patients. Our selected studies include 7 case series and 23 case reports. Most patients described were from the USA, followed by India and Iran. Tables 1, 2, and, 3 detail the data extracted from our selected studies.

Overall the age of the patients varied from 16 months to 82 years (Mode=64) and were predominantly males $(61.2 \% ; n=30)$. In $69.3 \%(n=34)$ of the cases, patients suffered from at least one comorbidity: $64.7 \%(\mathrm{n}=22)$ of them had arterial hypertension (men being affected twice as much as women), $52.9 \%(\mathrm{n}=18)$ had Diabetes mellitus, $17.6 \%(\mathrm{n}=6)$ had hyperlipidemia, $11.7 \%$ $(n=4)$ reported a previous myocardial infarction, and $8.8 \%(n=3)$ had a previous stroke. The duration of active COVID-19 infection varied from 3 to 90 days, with most cases lasting around 14 days. $97.9 \%(n=48)$ of all patients were hospitalized but only around $1 / 3$ of them were admitted into ICU (30.6\%). 32.65\% $(n=16)$ of the hospitalized patients required concurrent mechanical intubation/ventilation.

Regarding laboratory findings, $20.4 \%(n=10)$ of the total number of patients diagnosed with heart block had reduced left ventricular ejection fraction (LVEF) in the echocardiogram. Among these, LVEF was mildly reduced $(\mathrm{LVEF}=54-40 \%)$ in $50 \%(\mathrm{n}=5)$ and severely reduced $(\mathrm{LVEF}<35 \%)$ in $10 \%(\mathrm{n}=1)$. CRP was elevated in $55.4 \%(\mathrm{n}=27)$ of the patients, and $37 \%(\mathrm{n}=10)$ of them tested positive for HsCRP. WBC count was raised in $20.4 \%(n=10)$ of the patients. Myocardial involvement was measured in terms of increased Troponin I, which was raised in $22.4 \%(n=11)$ of the cases.

Our review found that $89.8 \%(\mathrm{n}=44)$ of the patients described in the included studies developed heart block during hospitalization for COVID-19 infection, while $10.20 \%(n=5)$ of the patients developed heart block following recovery. The type of heart block varied throughout the studies we reviewed, but the most common was third-degree heart block present in $61.22 \%(\mathrm{n}=30)$ of the patients. Junctional escape rhythm was seen in $30.61 \%(n=15)$, and Ventricular escape rhythm was seen in $26.53 \%(n=13)$ of the patients. The heart block was symptomatic in $53.06 \%(n=26)$, the most common symptom being dyspnea. 36.73\% $(n=18)$ of the patients showed spontaneous resolution of the heart block. On the other hand, 63.26\% $(n=31)$ of the patients had persistent heart block, out of which $16.33 \%(n=8)$ and $42.86 \%(n=21)$ were implanted with a temporary and permanent pacemaker, respectively. $24.49 \%(n=12)$ of the patients in the studies we reviewed were suspected of having myocarditis. However, none were confirmed with MRI or cardiac biopsy. The reported mortality rate in our study was $22.45 \% \quad(n=11)$ during hospitalization. We noted that $45.45 \%(n=5)$ of the patients who died had complete heart block ${ }^{11-}$ 
medRxiv preprint doi: https://doi.org/10.1101/2022.01.05.22268779; this version posted January 5, 2022. The copyright holder for this preprint (which was not certified by peer review) is the author/funder, who has granted medRxiv a license to display the preprint in perpetuity.

It is made available under a CC-BY 4.0 International license.

17. The other types of heart block reported in the other patients who died include Mobitz type 1 heart block with $\mathrm{RBBB}^{11}$, sinus pauses and sinus arrest, sinus tachycardia with an incomplete right bundle branch block $(\mathrm{RBBB})^{18}$, trifascicular block ${ }^{17}, \mathrm{RBBB}$ and left anterior fascicular block (LAFB), RBBB, left posterior fascicular block (LPFB) and incomplete trifascicular block $^{13}$.

\section{TABLE 1: Characteristics of included studies}

\begin{tabular}{|c|c|c|c|c|c|}
\hline$\underline{\text { ID }}$ & $\begin{array}{l}\text { Authors/Country/Study } \\
\text { Design }\end{array}$ & $\begin{array}{l}\text { Patient } \\
\text { number }\end{array}$ & Age & $\underline{\text { Sex }}$ & Co-morbidities \\
\hline 1 & $\begin{array}{l}\text { Babapoor-Farrokhran et } \\
\text { al./USA/Case Series }{ }^{19}\end{array}$ & 1 & 69 & Female & NA \\
\hline 2 & $\begin{array}{l}\text { Sardana et } \\
\text { al./USA/Case Report }{ }^{20}\end{array}$ & 1 & 49 & Female & Healthy \\
\hline 3 & $\begin{array}{lr}\text { Al-Assaf } & \text { et } \\
\text { al./UAE/Case } & \text { Report } \\
21(p) & \end{array}$ & 1 & 58 & Male & Hypertension \\
\hline \multirow[t]{7}{*}{4} & \multirow[t]{7}{*}{$\begin{array}{l}\text { Gupta et al./India/Case } \\
\text { Series }^{22}\end{array}$} & 1 & 55 & Female & Diabetes mellitus \\
\hline & & 2 & 56 & Female & NA \\
\hline & & 3 & 67 & Male & Diabetes mellitus, Hypertension \\
\hline & & 4 & 80 & Male & Hypertension, Prior Myocardial Infarction \\
\hline & & 5 & 45 & Female & NA \\
\hline & & 6 & 55 & Male & Prior Myocardial Infarction \\
\hline & & 7 & 69 & Male & Prior Heart Failure \\
\hline 5 & $\begin{array}{l}\text { Chinitz et al./USA/Case } \\
\text { Series }{ }^{16}\end{array}$ & Total 7 & $64 \pm 9.6$ & $\begin{array}{l}\text { males }(n=3) \\
\text { females }(n=4)\end{array}$ & $\begin{array}{l}\text { Hypertension, Diabetes mellitus, Obesity, } \\
\text { Hypercholesterolemia, Hepatic } \\
\text { disease/Cirrhosis, Cerebrovascular Disease / } \\
\text { Stroke prior to admission, Chronic Kidney } \\
\text { Disease }\end{array}$ \\
\hline
\end{tabular}


medRxiv preprint doi: https://doi.org/10.1101/2022.01.05.22268779; this version posted January 5, 2022. The copyright holder for this preprint (which was not certified by peer review) is the author/funder, who has granted medRxiv a license to display the preprint in perpetuity.

\section{It is made available under a CC-BY 4.0 International license .}

\begin{tabular}{|c|c|c|c|c|c|}
\hline \multirow[t]{2}{*}{6} & \multirow{2}{*}{$\begin{array}{l}\text { Kochav et al./USA/Case } \\
\text { Series }{ }^{11}\end{array}$} & \multirow[t]{2}{*}{1} & \multirow{2}{*}{$\begin{array}{l}76 \\
70\end{array}$} & \multirow{2}{*}{$\begin{array}{l}\text { Male } \\
\text { Male }\end{array}$} & \multirow{2}{*}{$\begin{array}{l}\text { Diabetes mellitus, Hypertension } \\
\text { Hypertension, Hyperlipidemia, Diabetes } \\
\text { mellitus, Ischemic cardiomiopathy }\end{array}$} \\
\hline & & & & & \\
\hline 7 & $\begin{array}{l}\text { Haddadin et } \\
\text { al./USA/Case Report }{ }^{23}\end{array}$ & 1 & 71 & Female & $\begin{array}{l}\text { Parkinson's disease, tardive dyskinesia, } \\
\text { Diabetes mellitus, bipolar disorder }\end{array}$ \\
\hline 8 & $\begin{array}{l}\text { Malekrah et } \\
\text { al./Iran/Case Report }{ }^{24}\end{array}$ & 1 & 71 & Male & NA \\
\hline 9 & $\begin{array}{l}\text { Ashok \& Loke/UK/Case } \\
\text { Report } 25\end{array}$ & 1 & 53 & Male & NA \\
\hline 10 & $\begin{array}{l}\text { Asli et al./Brunei/Case } \\
\text { Report }\end{array}$ & 1 & 60 & Female & Hypertension, hyperlipidemia, obesity \\
\hline \multirow[t]{2}{*}{11} & \multirow[t]{2}{*}{$\begin{array}{l}\text { He et al./China/Case } \\
\text { Series }{ }^{27}\end{array}$} & 1 & 66 & Female & NA \\
\hline & & 2 & 70 & Male & Hypertension, Diabetes mellitus \\
\hline 12 & $\begin{array}{l}\text { Dehghani Firouzabadi } \\
\text { et al./Iran/Case Report } \\
28\end{array}$ & 1 & 38 & Female & NA \\
\hline 13 & $\begin{array}{lr}\text { El-Assaad } & \text { et } \\
\text { al./USA/Case } & \text { Report } \\
29(p) & \end{array}$ & 1 & 10 & Male & Pityriasis lichenoides chronica \\
\hline \multirow[t]{3}{*}{14} & \multirow[t]{3}{*}{$\begin{array}{l}\text { Eneizat Mahdawi et } \\
\text { al./USA/Case Series }{ }^{12}\end{array}$} & 1 & 82 & Male & Hypertension, Stroke \\
\hline & & 2 & 55 & Male & NA \\
\hline & & 3 & 43 & Male & NA \\
\hline 15 & $\begin{array}{l}\text { Kir et al./ USA/Case } \\
\text { Report }^{30}\end{array}$ & 1 & 49 & Male & Mild intermittent asthma \\
\hline \multirow[t]{4}{*}{16} & \multirow[t]{4}{*}{$\begin{array}{l}\text { Dagher et al./USA/Case } \\
\text { Series }^{31}\end{array}$} & 1 & 42 & Female & Diabetes mellitus, Obesity \\
\hline & & 2 & 62 & Male & $\begin{array}{l}\text { Hypertension, Diabetes mellitus, coronary } \\
\text { artery disease,obesity }\end{array}$ \\
\hline & & 3 & 61 & Female & Hypertension,obesity \\
\hline & & 4 & 64 & Male & Hypertension,obesity \\
\hline 17 & $\begin{array}{l}\text { Domico et al./USA/Case } \\
\text { Report }^{32}\end{array}$ & 1 & 11 & Male & NA \\
\hline
\end{tabular}


medRxiv preprint doi: https://doi.org/10.1101/2022.01.05.22268779; this version posted January 5, 2022. The copyright holder for this preprint (which was not certified by peer review) is the author/funder, who has granted medRxiv a license to display the preprint in perpetuity.

\section{It is made available under a CC-BY 4.0 International license.}

\begin{tabular}{|c|c|c|c|c|c|}
\hline 18 & $\begin{array}{l}\text { Bahaidarah et al./Saudi } \\
\text { Arabia/Case Report }{ }^{17}\end{array}$ & 1 & 1.3 & Male & $\begin{array}{l}\text { Malnutrition, Persistent pulmonary } \\
\text { hypertension, Large mid-muscular VSD with } \\
\text { percutaneous closure }\end{array}$ \\
\hline 19 & $\begin{array}{l}\text { Gubitosa et } \\
\text { al./USA/Case Report }{ }^{13}\end{array}$ & 1 & 74 & Female & Diabetes mellitus \\
\hline 20 & $\begin{array}{l}\text { Aa et } \text { al./SA/Case } \\
\text { Report }^{33}\end{array}$ & 1 & 35 & Male & NA \\
\hline 21 & $\begin{array}{l}\text { Hosseini et } \\
\text { al./Iran/Case Report }\end{array}$ & 1 & 48 & Male & NA \\
\hline 22 & $\begin{array}{l}\text { Jean-Louis et } \\
\text { al./USA/Case Report }\end{array}$ & 1 & 60 & Male & $\begin{array}{l}\text { Hypertension, nonischemic dilated } \\
\text { cardiomyopathy, COPD, hyperlipidemia and } \\
\text { marijuana/cocaine abuse }\end{array}$ \\
\hline 23 & $\begin{array}{l}\text { Nikoo et al./Iran/Case } \\
\text { Report }{ }^{14}\end{array}$ & 1 & 38 & Female & $\begin{array}{l}\text { Previous history of mild covid infection } 2 \\
\text { months ago }\end{array}$ \\
\hline 24 & $\begin{array}{l}\text { Azarkish et } \\
\text { al./Iran/Case Report }\end{array}$ & 1 & 54 & Male & NA \\
\hline 25 & $\begin{array}{l}\text { Pecora et al./Italy/Case } \\
\text { Report }\end{array}$ & 1 & 54 & Female & NA \\
\hline 26 & $\begin{array}{l}\text { Maknojia et } \\
\text { al./USA/Case Report }\end{array}$ & 1 & 57 & Male & Hypertension \\
\hline 27 & $\begin{array}{l}\text { Jallad et al./USA/Case } \\
\text { Report }\end{array}$ & 1 & 71 & Male & Hypertension, Diabetes mellitus, RBBB \\
\hline 28 & Ali et al./USA/2021 ${ }^{38}$ & 1 & 70 & Male & NA \\
\hline 29 & $\begin{array}{l}\text { Ignatiuk et } \\
\text { al./Italy/Case Report }{ }^{39}\end{array}$ & 1 & 78 & Male & $\begin{array}{l}\text { Hypertension, Diabetes mellitus, } \\
\text { dyslipidemia, moderate aortic stenosis }\end{array}$ \\
\hline 30 & $\begin{array}{l}\text { Khalil et al./USA/Case } \\
\text { Report }\end{array}$ & 1 & 53 & Female & $\begin{array}{l}\text { End-stage-renal disease on peritoneal } \\
\text { dialysis, Anemia of chronic } \\
\text { disease,Hypertension,Diabetes mellitus }\end{array}$ \\
\hline
\end{tabular}




\section{TABLE 2: Outcome of the included patients}

\begin{tabular}{|c|c|c|c|c|}
\hline ID & Baseline ECG abnormality & Echo findings & $\begin{array}{l}\text { ICU } \\
\text { admitted }\end{array}$ & Death \\
\hline 1 & $\begin{array}{l}\text { Sinus arrhythmia, non-specific T-wave } \\
\text { abnormality, and prolonged QTC of } 474 \\
\text { ms. }\end{array}$ & Normal, LVEF>65\% & No & No \\
\hline 2 & NA & Normal, LVEF>65\% & NA & No \\
\hline 3 & $\begin{array}{l}\text { Initial ECG showed sinus bradycardia with } \\
\text { no ST-T wave changes and a repeat ECG } 1 \\
\text { hour later showed 2:1 second degree AV } \\
\text { block }\end{array}$ & Mildly dilated aescending aorta & No & No \\
\hline \multirow[t]{7}{*}{4} & NA & LVEF $=60 \%$ & No & No \\
\hline & NA & LVEF $=60 \%$ & No & No \\
\hline & NA & LVEF $=60 \%$ & No & No \\
\hline & NA & LVEF $=50 \%$ & No & No \\
\hline & NA & LVEF $=60 \%$ & No & No \\
\hline & NA & LVEF $=60 \%$ & No & No \\
\hline & NA & LVEF $=40 \%$ & No & No \\
\hline 5 & $\begin{array}{l}\text { RBBB } \quad(n=2), \text { LAHB + RBBB (n=1), 1st } \\
\text { degree AV block }(n=1) .\end{array}$ & $\begin{array}{l}\text { Normal left ventricular systolic function in } \\
\text { all patients ;Severe pulmonary HTN and/or } \\
\text { right ventricular dysfunction was identified } \\
\text { in } 29 \%\end{array}$ & $\begin{array}{l}1 \text { patient } \\
\text { admitted }\end{array}$ & Yes, $n=4$ \\
\hline \multirow[t]{2}{*}{6} & NA & Normal EF & Yes & Yes \\
\hline & NA & $\begin{array}{l}\text { LVEF decreased } 10 \% \text { to } 15 \% \text { with global } \\
\text { hypokinesis }\end{array}$ & Yes & Yes \\
\hline 7 & $\begin{array}{l}\text { Complete heart block with junctional } \\
\text { escape rythm }\end{array}$ & $\begin{array}{l}\text { LVEF70\%,normal biventricular wall motion, } \\
\text { thickened mitral valve leaflets, and dilated } \\
\text { left atrium. }\end{array}$ & NA & No \\
\hline 8 & $\begin{array}{l}\text { Second degree AV block with intermittent } \\
\text { left bundle branch block eventually } \\
\text { changing into Mobitz II type with fixed }\end{array}$ & Normal EF & No & No \\
\hline
\end{tabular}


medRxiv preprint doi: https://doi.org/10.1101/2022.01.05.22268779; this version posted January 5, 2022. The copyright holder for this preprint (which was not certified by peer review) is the author/funder, who has granted medRxiv a license to display the preprint in perpetuity.

It is made available under a CC-BY 4.0 International license .

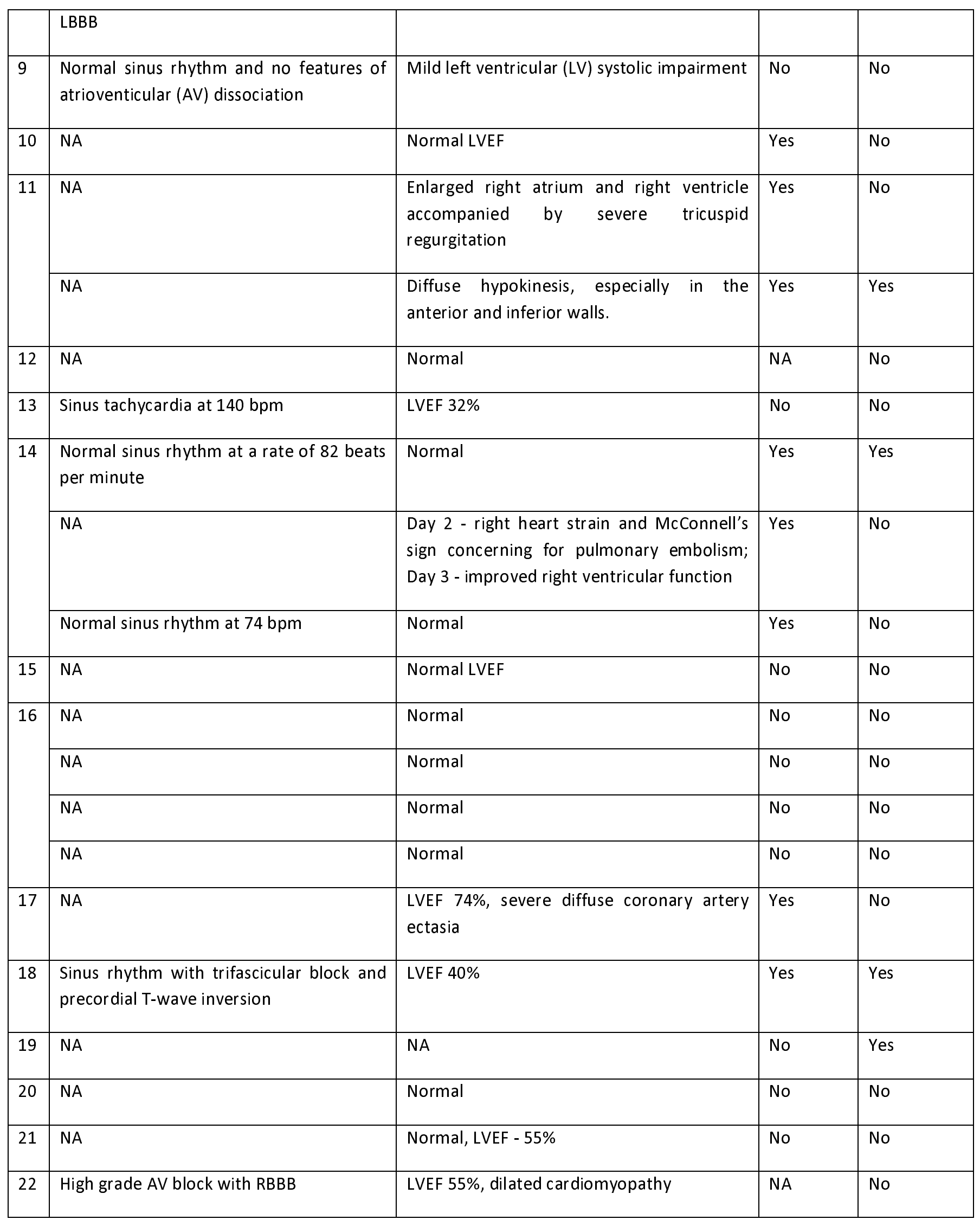


medRxiv preprint doi: https://doi.org/10.1101/2022.01.05.22268779; this version posted January 5, 2022. The copyright holder for this preprint (which was not certified by peer review) is the author/funder, who has granted medRxiv a license to display the preprint in perpetuity.

It is made available under a CC-BY 4.0 International license.

\begin{tabular}{|c|c|c|c|c|}
\hline 23 & NA & $\begin{array}{l}\text { LVEF } 20-25 \% \text {, Biventricular dilatation, Global } \\
\text { hypokinesia }\end{array}$ & No & No \\
\hline 25 & NA & $\begin{array}{l}\text { LVEF } 59 \% \text {, moderate regurgitation of mitral } \\
\text { valve }\end{array}$ & No & No \\
\hline 26 & NA & $\begin{array}{l}\text { normal-size left ventricle with a low LVEF - } \\
50 \%\end{array}$ & No & No \\
\hline 27 & $\begin{array}{l}\text { NSR, RBBB, Type } 2 \text { AV block and } \\
\text { Prolonged QT interval }\end{array}$ & $\begin{array}{l}\text { EF } 45 \% \text { and global hypokinesis with minor } \\
\text { regional wall motion abnormalities }\end{array}$ & Yes & No \\
\hline 29 & NA & $\begin{array}{l}\text { Nondilated hypertrophic left ventricle with } \\
\text { normal ejection fraction }\end{array}$ & Yes & No \\
\hline 30 & $\begin{array}{l}\text { Prolonged QT interval and complete AV } \\
\text { heart block. }\end{array}$ & $\begin{array}{l}\text { Normal LVEF, mild left atrial dilation, mild to } \\
\text { moderate AP and MT valvular regurgitation, } \\
\text { pericardial effusion, and a dilated ICV }\end{array}$ & Yes & No \\
\hline
\end{tabular}




\section{TABLE 3: Different types of heart block in the studied patients}

\begin{tabular}{|c|c|c|c|c|c|c|}
\hline ID & $\begin{array}{l}\text { Heart } \\
\text { block } \\
\text { during } \\
\text { COVID-19 } \\
\text { illness or } \\
\text { after } \\
\text { recovery? }\end{array}$ & $\begin{array}{l}\text { Self- } \\
\text { resolving } \\
\text { or } \\
\text { persistent }\end{array}$ & Type of heart block & $\begin{array}{l}\text { Escape } \\
\text { rhythm }\end{array}$ & $\begin{array}{l}\text { Was the } \\
\text { heart block } \\
\text { symptomatic }\end{array}$ & $\begin{array}{l}\text { Pacemaker } \\
\text { required? }\end{array}$ \\
\hline 1 & $\begin{array}{l}\text { during } \\
\text { illness }\end{array}$ & $\begin{array}{l}\text { self- } \\
\text { resolving }\end{array}$ & Mobitz type 1 & junctional & No & No \\
\hline 2 & $\begin{array}{l}\text { after } \\
\text { recovery }\end{array}$ & persistent & Intrahisian block & NA & Yes & permanent \\
\hline 3 & $\begin{array}{l}\text { during } \\
\text { illness }\end{array}$ & persistent & Mobitz type 1 & NA & No & permanent \\
\hline \multirow[t]{7}{*}{4} & $\begin{array}{l}\text { during } \\
\text { illness }\end{array}$ & persistent & Third degree & ventricular & Yes & permanent \\
\hline & $\begin{array}{l}\text { during } \\
\text { illness }\end{array}$ & persistent & Third degree & ventricular & Yes & permanent \\
\hline & $\begin{array}{l}\text { during } \\
\text { illness }\end{array}$ & persistent & Third degree & ventricular & Yes & permanent \\
\hline & $\begin{array}{l}\text { during } \\
\text { illness }\end{array}$ & persistent & Third degree & ventricular & Yes & permanent \\
\hline & $\begin{array}{l}\text { during } \\
\text { illness }\end{array}$ & $\begin{array}{l}\text { self- } \\
\text { resolving }\end{array}$ & Sick Sinus syndrome & junctional & Yes & No \\
\hline & $\begin{array}{l}\text { during } \\
\text { illness }\end{array}$ & persistent & Third degree & junctional & Yes & permanent \\
\hline & $\begin{array}{l}\text { during } \\
\text { illness }\end{array}$ & $\begin{array}{l}\text { self- } \\
\text { resolving }\end{array}$ & Sick Sinus syndrome & junctional & Yes & No \\
\hline 5 & $\begin{array}{l}\text { during } \\
\text { illness }\end{array}$ & persistent & $\begin{array}{l}\text { 2:1 AV block with preexisting RBBB(patient } \\
\text { 1) ,Sinus bradycardia and intermittent CHB } \\
\text { with junctional escape rhythm(patient } \\
\text { 2), CHB with wide complex escape rhythm } \\
\text { with preexisting LAHB + RBBB(patient 3), } \\
\text { CHB with narrow complex escape rhythm } \\
\text { with preexisting First degree AVB(patient }\end{array}$ & junctional & $\begin{array}{l}\text { symptomatic } \\
\text { in } 3 \text { patients }\end{array}$ & $\begin{array}{l}\text { temporary } \\
(n=2) \quad \text { and } \\
\text { permanent } \\
\text { pacemakers } \\
(n=5)\end{array}$ \\
\hline
\end{tabular}


medRxiv preprint doi: https://doi.org/10.1101/2022.01.05.22268779; this version posted January 5, 2022. The copyright holder for this preprint (which was not certified by peer review) is the author/funder, who has granted medRxiv a license to display the preprint in perpetuity.

It is made available under a CC-BY 4.0 International license .

\begin{tabular}{|c|c|c|c|c|c|c|}
\hline & & & 4), Sinus pauses(patients $5,6,7$ ), RBBB in 7 & & & \\
\hline \multirow[t]{2}{*}{6} & $\begin{array}{l}\text { during } \\
\text { illness }\end{array}$ & persistent & Mobitz type 1 and RBBB & junctional & Yes & No \\
\hline & $\begin{array}{l}\text { during } \\
\text { illness }\end{array}$ & persistent & $\begin{array}{l}\text { Day } 0 \text { - LBBB, Day } 5 \text { - complete heart block } \\
\text { (4) with no escape rhythm }\end{array}$ & NA & Yes & No \\
\hline 7 & $\begin{array}{l}\text { during } \\
\text { illness }\end{array}$ & persistent & Third degree & junctional & No & permanent \\
\hline 8 & $\begin{array}{l}\text { during } \\
\text { illness }\end{array}$ & persistent & $\begin{array}{l}\text { Day } 3 \text { - 2:1 AV block with intermittent } \\
\text { LBBB; Day } 4 \text { - 2:1 AV blockwoth fixed LBBB; } \\
\text { Day 6 - Atrial fibrillation with bradycardia } 1 \\
\text { month follow-up - Persistence of AV block }\end{array}$ & NA & No & permanent \\
\hline 9 & $\begin{array}{l}\text { during } \\
\text { illness }\end{array}$ & persistent & $\begin{array}{l}\text { Mobitz type } 2 \text { initially followed by Third } \\
\text { degree on the next day }\end{array}$ & junctional & Yes & permanent \\
\hline 10 & $\begin{array}{l}\text { during } \\
\text { illness }\end{array}$ & $\begin{array}{l}\text { self- } \\
\text { resolving }\end{array}$ & RBBB & NA & No & No \\
\hline \multirow[t]{2}{*}{11} & $\begin{array}{l}\text { during } \\
\text { illness }\end{array}$ & persistent & $\begin{array}{l}\text { Day } 0 \text { - 1st degree, Day of intubation - } \\
\text { Mobitz type 1, Next day - Third degree } \\
\text { [nearly complete AVB (or high-grade AVB) } \\
\text { developed], Soon after - revert to 1st } \\
\text { degree (SITIIIQIII disappeared) }\end{array}$ & Junctional & NA & No \\
\hline & $\begin{array}{l}\text { during } \\
\text { illness }\end{array}$ & persistent & $\begin{array}{l}\text { First ECG - sinus tachycardia with an } \\
\text { incomplete RBBB. }\end{array}$ & NA & No & No \\
\hline 12 & $\begin{array}{l}\text { after } \\
\text { recovery }\end{array}$ & $\begin{array}{l}\text { self- } \\
\text { resolving }\end{array}$ & Temporary complete heart block (4) & NA & Yes & No \\
\hline 13 & $\begin{array}{l}\text { during } \\
\text { illness }\end{array}$ & $\begin{array}{l}\text { self- } \\
\text { resolving }\end{array}$ & $\begin{array}{l}\text { 1st degree, Mobitz type 1, Mobitz type } 2 \text {, } \\
\text { Third degree (transient lasting for few } \\
\text { minutes) }\end{array}$ & junctional & No & No \\
\hline \multirow[t]{2}{*}{14} & $\begin{array}{l}\text { during } \\
\text { illness }\end{array}$ & persistent & $\begin{array}{l}\text { Day } 0 \text { - Complete Heart Block Day } 6 \text { [post } \\
\text { discontinuation of drugs] - Heart block } \\
\text { with narrow escape rhythm with } \\
\text { intermittent Wenckebach pattern and } \\
\text { escape-capture Wenckebach consistent } \\
\text { with AV nodal disease }\end{array}$ & ventricular & Yes & No \\
\hline & $\begin{array}{l}\text { during } \\
\text { illness }\end{array}$ & $\begin{array}{l}\text { self- } \\
\text { resolving }\end{array}$ & $\begin{array}{l}\text { Days 6-9 admission - Second degree AV } \\
\text { block with 2:1 conduction }\end{array}$ & NA & No & No \\
\hline
\end{tabular}


medRxiv preprint doi: https://doi.org/10.1101/2022.01.05.22268779; this version posted January 5, 2022. The copyright holder for this preprint (which was not certified by peer review) is the author/funder, who has granted medRxiv a license to display the preprint in perpetuity.

It is made available under a CC-BY 4.0 International license.

\begin{tabular}{|c|c|c|c|c|c|c|}
\hline & $\begin{array}{l}\text { during } \\
\text { illness }\end{array}$ & $\begin{array}{l}\text { self- } \\
\text { resolving }\end{array}$ & Third degree & NA & No & No \\
\hline 15 & $\begin{array}{l}\text { during } \\
\text { illness }\end{array}$ & $\begin{array}{l}\text { self- } \\
\text { resolving }\end{array}$ & Third degree & ventricular & Yes & temporary \\
\hline \multirow[t]{4}{*}{16} & $\begin{array}{l}\text { during } \\
\text { illness }\end{array}$ & $\begin{array}{l}\text { self- } \\
\text { resolving }\end{array}$ & Third degree & junctional & No & No \\
\hline & $\begin{array}{l}\text { during } \\
\text { illness }\end{array}$ & $\begin{array}{l}\text { self- } \\
\text { resolving }\end{array}$ & Third degree & ventricular & Yes & temporary \\
\hline & $\begin{array}{l}\text { during } \\
\text { illness }\end{array}$ & $\begin{array}{l}\text { self- } \\
\text { resolving }\end{array}$ & Third degree & ventricular & No & No \\
\hline & $\begin{array}{l}\text { during } \\
\text { illness }\end{array}$ & $\begin{array}{l}\text { self- } \\
\text { resolving }\end{array}$ & Third degree & junctional & No & No \\
\hline 17 & $\begin{array}{l}\text { after } \\
\text { recovery }\end{array}$ & $\begin{array}{l}\text { self- } \\
\text { resolving }\end{array}$ & $\begin{array}{l}\text { Early ECGs demonstrated normal sinus } \\
\text { rhythm.On day 4, he developed sinus } \\
\text { bradycardia with varying degrees of first } \\
\text { and second-degree heart block, } \\
\text { intraventricular conduction abnormalities } \\
\text { with varying QRS morphologies, and } \\
\text { periods of idioventricularrhythm.He had a } \\
\text { brief run of nonsustained ventricular } \\
\text { tachycardia. He then developed second } \\
\text { degree type II heart block with variable } \\
\text { nonspecific intraventricular conduction } \\
\text { delay.The following day, his conduction } \\
\text { system abnormalities improved, } \\
\text { progressing to second degree type I block. } \\
\text { Approximately } 48 \text { hours later, he was had } \\
\text { first-degree atrioventricular block.This was } \\
\text { followed by complete resolution of } \\
\text { conduction abnormalities, and a return to } \\
\text { normal sinus rhythm. }\end{array}$ & $\begin{array}{l}\text { junctional } \\
\text { and } \\
\text { ventricular }\end{array}$ & No & temporary \\
\hline 18 & $\begin{array}{l}\text { during } \\
\text { illness }\end{array}$ & persistent & Trifascicular block & NA & No & temporary \\
\hline 19 & $\begin{array}{l}\text { during } \\
\text { illness }\end{array}$ & persistent & $\begin{array}{l}\text { RBBB and LAFB, RBBB and LPFB, } 3 \\
\text { (incomplete trifascicular block) }\end{array}$ & NA & No & No \\
\hline 20 & $\begin{array}{l}\text { during } \\
\text { illness }\end{array}$ & persistent & Mobitz type 2 followed by Third degree & ventricular & No & $\begin{array}{l}\text { temporary, } \\
\text { followed by } \\
\text { permanent on }\end{array}$ \\
\hline
\end{tabular}


medRxiv preprint doi: https://doi.org/10.1101/2022.01.05.22268779; this version posted January 5, 2022. The copyright holder for this preprint (which was not certified by peer review) is the author/funder, who has granted medRxiv a license to display the preprint in perpetuity.

It is made available under a CC-BY 4.0 International license .

\begin{tabular}{|c|c|c|c|c|c|c|}
\hline & & & & & & day 18 \\
\hline 21 & $\begin{array}{l}\text { during } \\
\text { illness }\end{array}$ & persistent & Third degree & junctional & Yes & permanent \\
\hline 22 & $\begin{array}{l}\text { during } \\
\text { illness }\end{array}$ & $\begin{array}{l}\text { self- } \\
\text { resolving }\end{array}$ & $\begin{array}{l}\text { In October 2019, patient demonstrated } \\
\text { normal sinus rhythm (NSR) with a left } \\
\text { bundle branch block.Upon admission to } \\
\text { hospital in } 2020 \text {, he had a high-degree AV } \\
\text { block with a right bundle branch block } \\
\text { (RBBB) escape morphology. On day } 2 \text { of } \\
\text { hospitalization, his ECG had converted to } \\
\text { NSR with LBBB, which was consistent with } \\
\text { his ECG prior to this admission. He } \\
\text { remained without any recurrence of AV } \\
\text { block. }\end{array}$ & ventricular & Yes & No \\
\hline 23 & $\begin{array}{l}\text { after } \\
\text { recovery }\end{array}$ & $\begin{array}{l}\text { self- } \\
\text { resolving }\end{array}$ & $\begin{array}{l}\text { 4; After dexa the patient recovered to } 1 \\
\text { with LBBB; in } 4 \text { days it also resolved. }\end{array}$ & NA & Yes & temporary \\
\hline 24 & $\begin{array}{l}\text { during } \\
\text { illness }\end{array}$ & $\begin{array}{l}\text { self- } \\
\text { resolving }\end{array}$ & Third degree & NA & No & No \\
\hline 25 & $\begin{array}{l}\text { during } \\
\text { illness }\end{array}$ & persistent & $\begin{array}{l}\text { Left posterior hemiblock and high -grade } \\
\text { AV block }\end{array}$ & NA & Yes & temporary \\
\hline 26 & $\begin{array}{l}\text { during } \\
\text { illness }\end{array}$ & persistent & Third degree & ventricular & Yes & permanent \\
\hline 27 & $\begin{array}{l}\text { during } \\
\text { illness }\end{array}$ & persistent & Mobitz type 1 & NA & Yes & permanent \\
\hline 28 & $\begin{array}{l}\text { during } \\
\text { illness }\end{array}$ & $\begin{array}{l}\text { self- } \\
\text { resolving }\end{array}$ & Third degree & NA & Yes & permanent \\
\hline 29 & $\begin{array}{l}\text { during } \\
\text { illness }\end{array}$ & persistent & second degree 2:1 AV block; transient 4 & NA & No & permanent \\
\hline 30 & $\begin{array}{l}\text { after } \\
\text { recovery }\end{array}$ & persistent & Third degree & NA & Yes & permanent \\
\hline
\end{tabular}


medRxiv preprint doi: https://doi.org/10.1101/2022.01.05.22268779; this version posted January 5, 2022. The copyright holder for this preprint (which was not certified by peer review) is the author/funder, who has granted medRxiv a license to display the preprint in perpetuity.

It is made available under a CC-BY 4.0 International license .

\section{Changes in the type of heart block during the clinical course:}

We have found that $61.22 \%$ of the patients in our case studies developed a third-degree heart block. Some other ECG findings reported after COVID-19 infection include first-degree heart block ( $n=4)$; Mobitz type $1(n=7)$; Mobitz type $2(n=10)$; atrial fibrillation (AF) with bradycardia $(n=1)$; Intrahisian block $(n=1)$ and Trifasicular block $(n=1)$. Six patients experienced RBBB and four patients experienced LBBB. Sick sinus syndrome was also detected in 2 patients ${ }^{22}$. Many patients had changes in the type of heart block during the course of illness. Alabdulgader et al. described a patient who had persistent heart block and received a temporary pacemaker; this was later replaced with a permanent pacemaker on day 18 of hospitalization ${ }^{33}$. Pecora et al described a patient with left posterior hemiblock and high-grade AV block, which resolved with implantation of a temporary pacemaker ${ }^{36} .2$ patients developed Mobitz type 2 heart block, which was followed by 3rd-degree complete heart block ${ }^{13,41}$. Azakirsh et al illustrated a patient who developed Mobitz type 2 AV block (2:1) with intermittent LBBB on the 3rd day of hospitalization; this evolved on the fourth day to Mobitz type 2 AV block (2:1) with fixed $\mathrm{LBBB}$, and on the sixth day to AF with bradycardia. The persistence of AV block was observed one month after discharge ${ }^{15}$. Domico et al reported a child with a high-grade heart block, which resolved with temporary pacing. Upon admission, the patient's ECGs exhibited normal sinus rhythm. On the fourth day, the patient developed sinus bradycardia along with varying degrees of first and second-degree heart block, episodes of idioventricular rhythm, and intraventricular conduction abnormalities associated with fluctuating morphologies in QRS complexes. Consequently, the patient experienced a transient nonsustained ventricular tachycardia (VT) followed by a second-degree type II heart block with inconsistent nonspecific intraventricular conduction delay. On day 5, the irregularities of the conduction system improved and settled into a second-degree type I block. On day 7, he demonstrated a first-degree AV block which was followed by complete resolution of conduction abnormalities and return to sinus rhythm ${ }^{32}$.

One patient showed inferior and precordial leads ST-segment elevation on day 35 of hospitalization. In addition, there was a progressive increase in ST-elevation amplitude which transformed into a triangular QRS-ST-T waveform. Two attacks of multifocal ventricular tachycardia were observed during the development of ST-segment elevation ${ }^{27}$.

Additional ECG findings reported in the included studies are sinus bradycardia ${ }^{19}$, sinus tachycardia $^{26}$, sinus pauses in 3 patients ${ }^{16}$, and QT prolongation ${ }^{24,32,37,40}$.

\section{DISCUSSION}

Every day brings about a new discovery about the pathogenesis and clinical manifestations of COVID-19. According to the fast-growing availability of scientific literature on this topic, the heart appears to be one of the elective targets of the virus. It is known that SARS-CoV enters into human cells via Angiotensin-Converting Enzyme 2 (ACE-2) receptors which are found in alveolar epithelial cells and endothelium of arteries and veins. Multiple hypotheses have been made to explain the mechanism of heart blocks in COVID-19 infection. In autopsy studies, SARS-CoV-2 virus and inflammatory infiltrate have been found in the myocardium, which implies direct viral invasion of the heart. ACE-2 downregulation decreases the action of angiotensin 1-7 leading to increased synthesis of inflammatory mediators like TNF $\alpha, \mathrm{CRP}$, and TGF $\beta$ which produces a cytokine storm. TGF $\beta$ induces interstitial fibrosis, which damages 
medRxiv preprint doi: https://doi.org/10.1101/2022.01.05.22268779; this version posted January 5, 2022. The copyright holder for this preprint (which was not certified by peer review) is the author/funder, who has granted medRxiv a license to display the preprint in perpetuity.

It is made available under a CC-BY 4.0 International license.

cardiac architecture. Troponin elevation and contractility dysfunction occur in the setting of severe hypoxia due to inflammatory damage or hypercoagulability. These phenomena initiated by the virus can occur in parallel with direct viral damage and interact with each other, enhancing their effect ${ }^{8,42}$.

In our study, only $24.49 \%$ of the patients were suspected of myocarditis. However, none were confirmed with MRI or cardiac biopsy. Kir et al. reported one patient with no evidence of myocardial involvement indicated by normal levels of cardiac enzymes and no findings on ECG, who developed a self-resolving 3rd-degree heart block. It is postulated that COVID-19 triggered subclinical myocarditis may have given rise to high-degree AV block in this patient ${ }^{30}$. The mechanism of heart block in other cases is poorly understood. In our review, all the patients who died after developing heart block were on mechanical ventilation in the intensive care unit.

Deterioration of any pre-existing diseases in the conduction system such as AV node disease, bundle branch blocks, or His-Purkinje system disorder can cause new advanced blocks, leading to poor clinical outcomes ${ }^{34}$. Another big role in COVID-19 progression is played by comorbidities and risk factors. Cardiovascular diseases, hypertension, diabetes mellitus, renal disease, liver disease, cerebrovascular disease, obesity, hyperlipidemia, and smoking history have a crucial impact on disease progression and complications ${ }^{8}$. In our review, hypertension and diabetes mellitus were present in the majority of patients who passed away due to heart block during COVID-19 infection. Since many of these risk factors are modifiable, lifestyle changes, early diagnosis, and management of comorbidities must be considered for better outcomes in COVID-19 patients.

Pathophysiological, histological, and imaging data ${ }^{8,43}$ indicate that SARS-CoV-2 could induce tissue damage, which would predispose patients to recurrent cardiac issues long-term after discharge. However, the long-term impact of COVID-19 induced heart blocks on late cardiac manifestations are not well studied, thus leading to poor clinical guidance regarding remote cardiac follow-up after discharge.

\section{$\underline{\text { Limitations }}$}

One of the limitations of our systematic review was the restricted number of studies and size of the sample population. We have only included studies published until May 2021. Future reports of heart block in COVID-19 patients might reveal new relations between the severity of the infection and the prognosis of patients who develop heart block.

The resolution of ECGs changes after discontinuation of HCQ in a case suggests a causal relationship between hydroxychloroquine and ECG abnormalities. Thus the role of certain medications, like hydroxychloroquine, on the development of arrhythmias in the presence of COVID-19 infection must also be further investigated ${ }^{26}$.

Also, the presence of COVID-19 induced myocarditis was not confirmed via biopsy or MRI in any of the patients who developed heart block in our included studies. 
medRxiv preprint doi: https://doi.org/10.1101/2022.01.05.22268779; this version posted January 5, 2022. The copyright holder for this preprint (which was not certified by peer review) is the author/funder, who has granted medRxiv a license to display the preprint in perpetuity.

It is made available under a CC-BY 4.0 International license.

\section{Conclusion}

The exact pathogenesis of heart block in COVID-19 patients must be further explored. More research is required to determine whether myocarditis due to COVID-19 is associated with the development of heart blocks. It is also needed to be studied further whether heart block is an independent risk factor for a worse prognosis in COVID-19 cases.

Patients hospitalized for COVID-19 must be continuously monitored for arrhythmias including heart blocks, especially in the presence of comorbidities. Physicians must be made aware of these cardiac manifestations of the infection, as early detection can shorten the hospital stay and improve the prognosis of the patient.

\section{Contributions:}

MRA contributed to research idea, design of the study, collecting data, statistical analysis and interpretation, writing the first draft, writing and editing the final manuscript. MSh contributed to study design, data extraction, statistical analysis and interpretation, writing the first draft. MS, CS, VR and NS contributed to data extraction, statistical analysis \& interpretation and writing the first manuscript.

Corresponding Author: Correspondence to Md Ripon Ahammed

\section{Conflict of Interest: None}

Acknowledgment: To Larkin Community Hospital, FL, USA for supporting and providing our research platform.

Funding: None

Ethical Approval: Not applicable

\section{$\underline{\text { References: }}$}

1. Wang D, Hu B, Hu C, et al. Clinical Characteristics of 138 Hospitalized Patients With 2019 Novel Coronavirus-Infected Pneumonia in Wuhan, China. JAMA. 2020;323(11):1061-1069. doi:10.1001/jama.2020.1585

2. Gupta A, Madhavan MV, Sehgal K, et al. Extrapulmonary manifestations of COVID-19. Nat Med. 2020;26(7):1017-1032. doi:10.1038/s41591-020-0968-3 
medRxiv preprint doi: https://doi.org/10.1101/2022.01.05.22268779; this version posted January 5, 2022. The copyright holder for this preprint (which was not certified by peer review) is the author/funder, who has granted medRxiv a license to display the preprint in perpetuity.

It is made available under a CC-BY 4.0 International license .

3. Sabatino J, Rosa SD, Salvo GD, Indolfi C. Impact of cardiovascular risk profile on COVID19 outcome. A meta-analysis. PLOS ONE. 2020;15(8):e0237131. doi:10.1371/journal.pone.0237131

4. Creel-Bulos C, Hockstein M, Amin N, Melhem S, Truong A, Sharifpour M. Acute Cor Pulmonale in Critically Ill Patients with Covid-19. N Engl J Med. 2020;382(21):e70. doi:10.1056/NEJMc2010459

5. Inciardi RM, Lupi L, Zaccone G, et al. Cardiac Involvement in a Patient With Coronavirus Disease $2019 \quad$ (COVID-19). JAMA Cardiol. 2020;5(7):819-824. doi:10.1001/jamacardio.2020.1096

6. Singh S, Desai R, Gandhi Z, et al. Takotsubo Syndrome in Patients with COVID-19: a Systematic Review of Published Cases. SN Compr Clin Med. Published online October 6, 2020:1-7. doi:10.1007/s42399-020-00557-w

7. Hua A, O'Gallagher K, Sado D, Byrne J. Life-threatening cardiac tamponade complicating myo-pericarditis in COVID-19. Eur Heart J. 2020;41(22):2130. doi:10.1093/eurheartj/ehaa253

8. Babapoor-Farrokhran S, Gill D, Walker J, Rasekhi RT, Bozorgnia B, Amanullah A. Myocardial injury and COVID-19: Possible mechanisms. Life Sciences. 2020;253:117723. doi:10.1016/j.lfs.2020.117723

9. Malaty M, Kayes T, Amarasekera AT, Kodsi M, MacIntyre CR, Tan TC. Incidence and treatment of arrhythmias secondary to coronavirus infection in humans: A systematic review. Eur J Clin Invest. 2021;51(2):e13428. doi:10.1111/eci.13428

10. Garcia-Zamora S, Lee S, Haseeb S, et al. Arrhythmias and electrocardiographic findings in Coronavirus disease 2019: A systematic review and meta-analysis. Pacing and Clinical Electrophysiology. 2021;44(6):1062-1074. doi:10.1111/pace.14247

11. Kochav SM, Coromilas E, Nalbandian A, et al. Cardiac Arrhythmias in COVID-19 Infection. Circ Arrhythm Electrophysiol. 2020;13(6):e008719. doi:10.1161/CIRCEP.120.008719

12. Eneizat Mahdawi T, Wang H, Haddadin FI, Al-Qaysi D, Wylie JV. Heart block in patients with coronavirus disease 2019: A case series of 3 patients infected with SARS-CoV-2. HeartRhythm Case Rep. 2020;6(9):652-656. doi:10.1016/j.hrcr.2020.06.014

13. Gubitosa JC, Xu P, Ahmed A, Pergament K. Incomplete Trifascicular Block and Mobitz Type II Atrioventricular Block in COVID-19. Cureus. 2020;12(9):e10461. doi:10.7759/cureus.10461

14. Nikoo MH, Mozaffari R, Hatamnejad MR, Bazrafshan M, Kasaei M, Bazrafshan H. Systolic dysfunction and complete heart block as complications of fulminant myocarditis in a recovered COVID-19 patient. J Cardiol Cases. Published online April 20, 2021. doi:10.1016/j.jccase.2021.03.009 
medRxiv preprint doi: https://doi.org/10.1101/2022.01.05.22268779; this version posted January 5, 2022. The copyright holder for this preprint (which was not certified by peer review) is the author/funder, who has granted medRxiv a license to display the preprint in perpetuity.

It is made available under a CC-BY 4.0 International license .

15. Azarkish M, Laleh Far V, Eslami M, Mollazadeh R. Transient complete heart block in a patient with critical COVID-19. Eur Heart J. 2020;41(22):2131. doi:10.1093/eurheartj/ehaa307

16. Chinitz JS, Goyal R, Harding M, et al. Bradyarrhythmias in patients with COVID-19: Marker of poor prognosis? Pacing Clin Electrophysiol. 2020;43(10):1199-1204. doi:10.1111/pace. 14042

17. Bahaidarah SA, Dohain AM, Abdelmohsen G, Alnajjar AA, Al-Ata J. Impact of coronavirus disease 2019 in a child who underwent ventricular septal defect device closure: a case report. European Heart Journal - Case Reports. 2021;5(1). doi:10.1093/ehjcr/ytaa478

18. Complete Heart Block: A Rare Presentation of COVID-19. HMP Global Learning Network. Published May 3, 2021. Accessed September 21, 2021. https://www.hmpgloballearningnetwork.com/site/cathlab/content/complete-heart-blockrare-presentation-covid-19

19. Babapoor-Farrokhran S, Batnyam U, Wiener PC, et al. Atrioventricular and Sinus Node Dysfunction in Stable COVID-19 Patients. SN Compr Clin Med. Published online September 4, 2020:1-4. doi:10.1007/s42399-020-00497-5

20. Sardana M, Scheinman MM, Moss JD. Atrioventricular block after COVID-19: What is the mechanism, site of block, and treatment? Heart Rhythm. 2021;18(3):489-490. doi:10.1016/j.hrthm.2020.10.020

21. Al-Assaf O, Mirza M, Musa A. Atypical presentation of COVID-19 as subclinical myocarditis with persistent high-degree atrioventricular block treated with pacemaker implant. HeartRhythm Case Rep. 2020;6(11):884-887. doi:10.1016/j.hrcr.2020.09.003

22. Gupta MD, Qamar A, Mp G, et al. Bradyarrhythmias in patients with COVID-19: A case series. Indian Pacing Electrophysiol J. 2020;20(5):211-212. doi:10.1016/j.ipej.2020.08.004

23. Haddadin FI, Mahdawi TE, Hattar L, Beydoun H, Fram F, Homoud M. A case of complete heart block in a COVID-19 infected patient. J Cardiol Cases. 2020;23(1):27-30. doi:10.1016/j.jccase.2020.08.006

24. Malekrah A, Fatahian A. A case report of a rare cardiac complication in novel coronavirus disease. Eur Heart J Case Rep. 2020;4(6):1-4. doi:10.1093/ehjcr/ytaa323

25. Ashok V, Loke WI. Case report: high-grade atrioventricular block in suspected COVID-19 myocarditis. Eur Heart J Case Rep. 2020;4(FI1):1-6. doi:10.1093/ehjcr/ytaa248

26. Asli R, Abdullah MS, Chong PL, et al. Case Report: Right Bundle Brunch Block and QTc Prolongation in a Patient with COVID-19 Treated with Hydroxychloroquine. Am J Trop Med Hyg. 2020;103(1):79-82. doi:10.4269/ajtmh.20-0376 
medRxiv preprint doi: https://doi.org/10.1101/2022.01.05.22268779; this version posted January 5, 2022. The copyright holder for this preprint (which was not certified by peer review) is the author/funder, who has granted medRxiv a license to display the preprint in perpetuity.

It is made available under a CC-BY 4.0 International license.

27. He J, Wu B, Chen Y, et al. Characteristic Electrocardiographic Manifestations in Patients With COVID-19. Can J Cardiol. 2020;36(6):966.e1-966.e4. doi:10.1016/j.cjca.2020.03.028

28. Dehghani Firouzabadi M, Goudarzi S, Dehghani Firouzabadi F, Moosaie F. Complete heart block and itchy rash in a patient with COVID-19. Caspian J Intern Med. 2020;11(Suppl 1):569-571. doi:10.22088/cjim.11.0.569

29. El-Assaad I, Hood-Pishchany MI, Kheir J, et al. Complete Heart Block, Severe Ventricular Dysfunction, and Myocardial Inflammation in a Child With COVID19 Infection. JACC Case Rep. 2020;2(9):1351-1355. doi:10.1016/j.jaccas.2020.05.023

30. Kir D, Mohan C, Sancassani R. Heart Brake: An Unusual Cardiac Manifestation of COVID-19. JACC Case Rep. 2020;2(9):1252-1255. doi:10.1016/j.jaccas.2020.04.026

31. Dagher L, Wanna B, Mikdadi G, Young M, Sohns C, Marrouche NF. High-degree atrioventricular block in COVID-19 hospitalized patients. Europace. 2021;23(3):451-455. doi:10.1093/europace/euaa333

32. Domico M, McCanta AC, Hunt JL, Ashouri N, Nugent D, Kelly RB. High-grade heart block requiring transvenous pacing associated with multisystem inflammatory syndrome in children during the COVID-19 pandemic. HeartRhythm Case Rep. 2020;6(11):811-814. doi:10.1016/j.hrcr.2020.08.015

33. Aa A, A A, M S, H E, K AK. Novel Behavior of the 2019 Novel Coronavirus With Invasion of the Cardiac Conduction System in the Young. Cureus. 2020;12(10). doi:10.7759/cureus. 11115

34. Hosseini Z, Ghodsi S, Hejazi SF. Persistent Complete Heart Block in a Patient with COVID-19 Infection: a Case Report. SN Compr Clin Med. Published online January 6, 2021:1-4. doi:10.1007/s42399-020-00712-3

35. Jean-Louis F, Adedayo AM, Ajibawo T, Upadhya G, Syed A. A Rare Case of Resolution of High-Degree Atrioventricular Block Associated With COVID-19. J Med Cases. 2020;11(8):243-245. doi:10.14740/jmc3524

36. Pecora D, La Greca C, Pezzotti E, Botti P, Campana M, Cuccia C. [An unusual presentation of cardiac involvement during the COVID-19 pandemic]. G Ital Cardiol (Rome). Published online 2020:594-597.

37. Jallad A, Doodnauth AV, Lee J, et al. New Onset, Irreversible, Prolonged QT-Interval Requiring Permanent Biventricular Pacemaker in a COVID-19 Patient. Am J Med Case Rep. 2021;9(4):249-252. doi:10.12691/ajmcr-9-4-11

38. Ali AA, Maaliki N, Omar M, Roemer A, Ruiz J, Esmail K. Transient Third-degree Heart Block Secondary to Asymptomatic COVID-19 Infection. American Journal of Medical Case Reports. 2021;9(4):259-260. doi:10.12691/ajmcr-9-4-13 
medRxiv preprint doi: https://doi.org/10.1101/2022.01.05.22268779; this version posted January 5, 2022. The copyright holder for this preprint (which was not certified by peer review) is the author/funder, who has granted medRxiv a license to display the preprint in perpetuity.

It is made available under a CC-BY 4.0 International license.

39. Ignatiuk B, Baratto F, Monticelli J, Bacchion F, Marchese GM, Pasquetto G. Pacemaker implantation in a COVID $\square 19$ patient. Balancing the patient's needs and the team's risk of exposure. J Arrhythm. 2020;37(1):261-263. doi:10.1002/joa3.12480

40. Khalil S, Collins A, Mubarak N, Khalil T. Third degree heart block and elevated troponin following acute respiratory hypoxic failure in a COVID-19 recovered patient in the absence of coronary artery disease. J Cardio Case Rep. 2020;4(2). doi:10.15761/JCCR.1000159

41. John T-J, Kyriakakis C, Zachariah D, Doubell A. Inferior ST-elevation myocardial infarction managed with a pharmacoinvasive strategy and conservative management of delayed atrioventricular block: classical case report. Eur Heart J Case Rep. 2020;4(6):1-7. doi:10.1093/ehjcr/ytaa375

42. Liu PP, Blet A, Smyth D, Li H. The Science Underlying COVID-19: Implications for the Cardiovascular System.

Circulation. 2020;142(1):68-78. doi:10.1161/CIRCULATIONAHA.120.047549

43. Rajpal S, Tong MS, Borchers J, et al. Cardiovascular Magnetic Resonance Findings in Competitive Athletes Recovering From COVID-19 Infection. JAMA Cardiol. 2021;6(1):116-118. doi:10.1001/jamacardio.2020.4916 